ARTICLE

DOI: $10.1038 / s 41467-017-00068-8$

OPEN

\title{
Two-step synthesis of chiral fused tricyclic scaffolds from phenols via desymmetrization on nickel
}

Ravindra Kumar ${ }^{1}$, Yoichi Hoshimoto (1) ${ }^{1,2}$, Eri Tamai ${ }^{1}$, Masato Ohashi ${ }^{1}$ \& Sensuke Ogoshi (i) ${ }^{1}$

Tricyclic furan derivatives with multiple chiral centers are ubiquitous in natural products. Construction of such tricyclic scaffolds in a stereocontrolled, step-economic, and atom-economic manner is a key challenge. Here we show a nickel-catalyzed highly enantioselective synthesis of hydronaphtho[1,8-bc]furans with five contiguous chiral centers via desymmetrization of alkynyl-cyclohexadienone by oxidative cyclization and following formal [4+2] cycloaddition processes. Alkynyl-cyclohexadienone was synthesized in one step from easily accessible phenols. This reaction represents excellent chemo-selectivity, regio-selectivity, diastereo-selectivity, and enantio-selectivity (single diastereomer, up to $99 \%$ ee). An extraordinary regioselectivity in the formal [4+2] cycloaddition step with enones revealed the diverse reactivity of the nickelacycle intermediate. Desymmetrization of alkynyl-cyclohexadienones via oxidative cyclization on nickel was supported by the isolation of a nickelacycle from a stoichiometric reaction. Enantioenriched tricyclic products contain various functional groups such as $\mathrm{C}=\mathrm{O}$ and $\mathrm{C}=\mathrm{C}$. The synthetic utility of these products was demonstrated by derivatization of these functional groups.

\footnotetext{
${ }^{1}$ Department of Applied Chemistry, Faculty of Engineering, Osaka University, Suita, Osaka 565-0871, Japan. ${ }^{2}$ Frontier Research Base for Global Young Researchers, Graduate School of Engineering, Osaka University, Suita, Osaka 565-0871, Japan. Correspondence and requests for materials should be addressed to S.O. (email: ogoshi@chem.eng.osaka-u.ac.jp)
} 
ydronaphtho[1,8-bc]furan rings with multiple chiral centers are a common structural motif in biologically active natural products (Fig. 1a) ${ }^{1-5}$. Such tricyclic structures are also found in key synthetic intermediates that are employed in a large number of sesquiterpenoids ${ }^{6-9}$. Owing to diverse biological activities and synthetic potentials associated with these fused tricyclic structures, a significant amount of attention has been paid to their enantioselective syntheses. Despite the existence of various stepwise stereoselective methods ${ }^{1-9}$, direct access to such tricyclic fused rings in a completely enantio-controlled, diastereo-controlled, step-economic, and atom-economic manner would be a remarkable development ${ }^{10-13}$. Recently, Alemán reported a straightforward method for the construction of tricyclic fused rings from a cyclohexadienone tethered alkenal by employing an organocatalyzed asymmetric desymmetrization strategy (Fig. 1b) ${ }^{14}$. In this process, desymmetrization step involved the intramolecular $[4+2]$ cycloaddition of chiral dienamine with a diastereotropic enone constructed tricyclic fused rings with three chiral centers.

We envisaged the enantioselective desymmetrization ${ }^{15-19}$ of alkynyl-cyclohexadienone via an intramolecular oxidative cyclization on nickel in the presence of a chiral ligand. Alkynylcyclohexadienone were synthesized in one step from easily accessible phenols. The oxidative cyclization of an enantiotropic enone with a tethered alkyne unit would form a tricyclic fused nickelacycle ${ }^{20-24}$ with three chiral centers, which could react with another olefin to yield a tricyclic product with the concomitant generation of two more chiral centers. Nickel( 0$)$-catalyzed trimerization of an alkyne with two enones has been reported by Montgomery et al. ${ }^{25-28}$ and by us ${ }^{29,30}$. Here, we report a nickel(0)-catalyzed enantioselective synthesis of chiral hydronaphtho[1,8-bc]furans with five contiguous chiral centers via desymmetrization of alkynyl-cyclohexadienone and following intermolecular formal [4+2] cycloaddition reaction processes.

\section{Results}

Reaction optimization. Prior to developing the reaction in an asymmetric fashion, achiral ligands were examined using cyclohexadienone-yne (1a) and 4-methoxychalcone (2a) for the model transformation to hydronaphtho[1,8-bc] furan 3aa (Fig. 2, see also Supplementary Table 1 for detail). 1,3-Bis-(2,6-diisopropylphenyl)imidazol-2-ylidene (IPr) proved to be an optimal ligand to deliver rac-3aa in $96 \%$ yield, whereas $\mathrm{PCy}_{3}$ failed to give any product. A single diastereomer of 3aa was obtained out of sixteen possible isomers. Moreover, it is remarkable that an extraordinary regioselectivity was observed in the formal $[4+2]$ cycloaddition step, fixing two carbonyls at the 1,4-positions in 3aa, whereas 1,5-dicarbonyl compounds were obtained in reports of nickel( 0$)$-catalyzed cycloaddition reactions ${ }^{20-30}$. Considering a

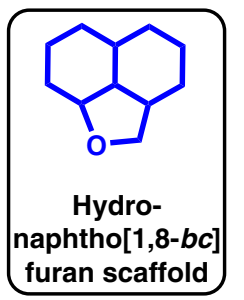

(1)

$(-)$-Teucvidin (antitumor)

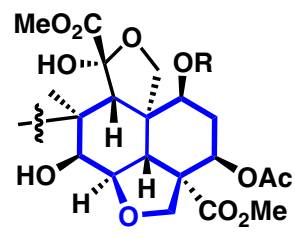

$(-)$-Azadirachtin fragment (insecticide)

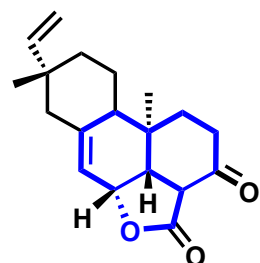

Momilactone A (allelochemical)

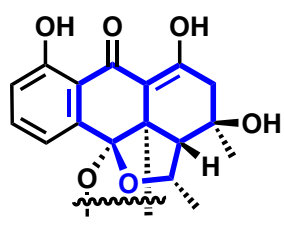

(-)-Bisanthraquinone fragment (antitumor)

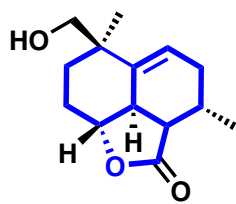

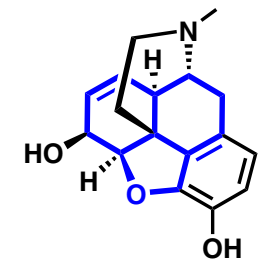

(-)-Morphine (analgesic)<smiles>CC1=C2C(=O)O[C@H]3CCC(C)(C)[C@]2(C)[C@]32OC(C)(C)O[C@H]12</smiles>

Ziegler intermediate for (-)-forskolin

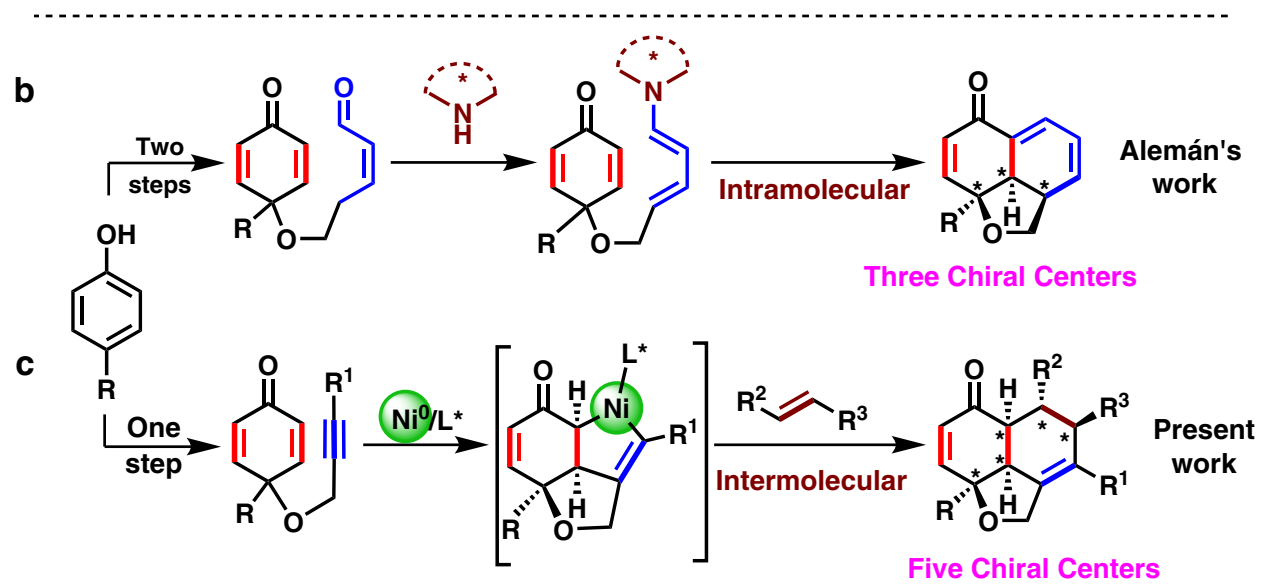

Fig. 1 Hydronaphtho[1,8-bc]furans and their syntheses from phenols. a Representative examples of biologically active compounds. b Previous work employed desymmetrization by intramolecular [4+2] cycloaddition, catalyzed by organocatalyst. c Present work employed desymmetrization by oxidative cyclization and following intermolecular formal [4+2] cycloaddition process with nickel 


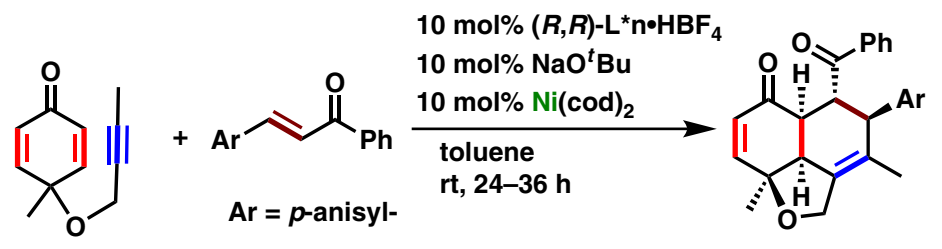

1a $2 a$

$(S, S, R, R, S)-3$ aa
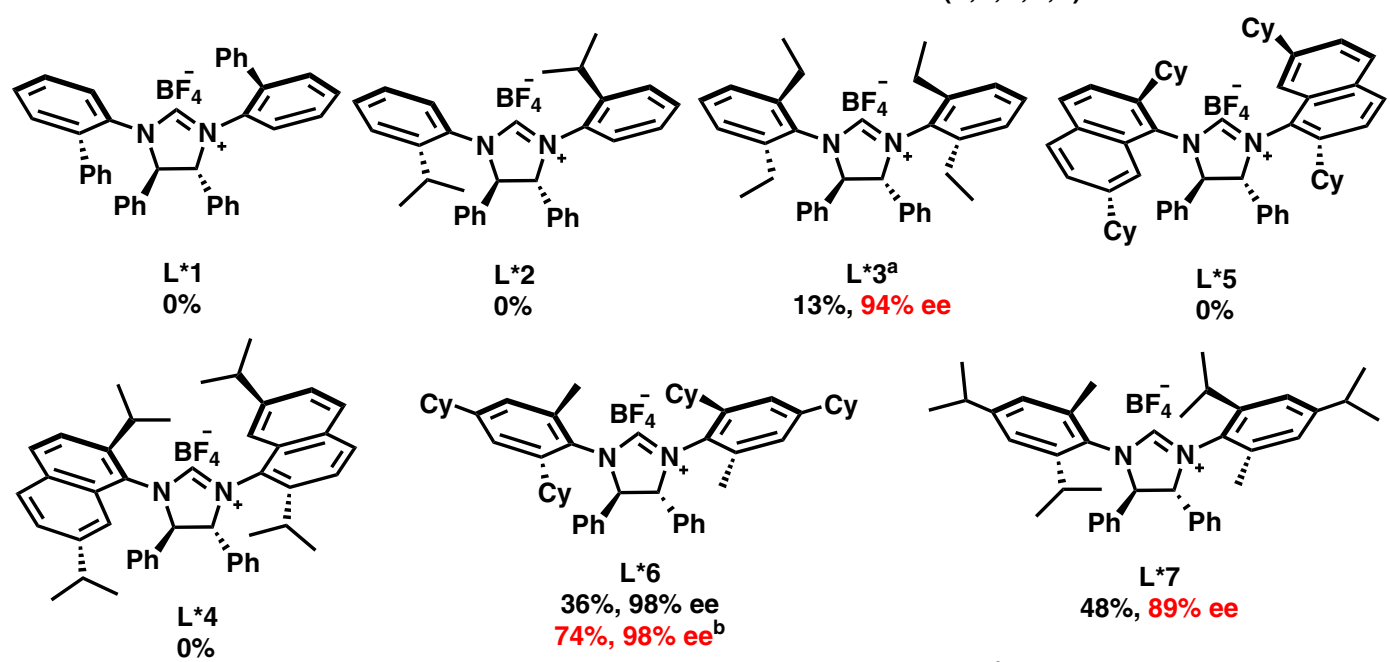

$36 \%, 98 \%$ ee

$74 \%, 98 \%$ ee $^{b}$

L*7

$\operatorname{IPr}($ Rac. 3aa; 96\%)

$\mathrm{PCy}_{3}(0 \%)^{\mathrm{d}}$

Fig. 2 Evaluation of Ligands. $\left.(R, R)-\mathbf{L}^{\star} \mathbf{n} \cdot \mathrm{HBF}_{4}, \mathrm{NaO}^{\mathrm{t}} \mathrm{Bu}, \mathrm{Ni}(\mathrm{cod})\right)_{2}(0.01 \mathrm{mmol}$ each), 1a $(0.12 \mathrm{mmol}), \mathbf{2 a}(0.10 \mathrm{mmol})$, and toluene $(1.0 \mathrm{ml})$ are employed. Isolated yields are given and enantioselectivity was determined by SFC equipped with a chiral stationary phase. ${ }^{\text {a }}$ ield and enantioselectivity was measured at $27 \%$ conversion in 3 days. ${ }^{b} \mathrm{ml}$ toluene was employed and reaction was conducted at $60^{\circ} \mathrm{C} .{ }^{\mathrm{C}} 10 \mathrm{~mol} \% \mathrm{IPr}$ was used instead of chiral $\mathrm{NHC}$ salt and $\mathrm{NaO}^{t} \mathrm{Bu} .{ }^{\mathrm{d}} 2 \mathrm{O}$ mol\% $\mathrm{PCy}_{3}$ was used instead of chiral $\mathrm{NHC}$ salt and $\mathrm{NaO}^{t} \mathrm{Bu}$

the efficiency of $\mathrm{N}$-heterocyclic carbene (NHC) in this transformation, chiral $(R, R)$-NHCs, generated in situ by treating the corresponding imidazolinium salts with $\mathrm{NaO}^{t} \mathrm{Bu}$, were investigated to afford enantioenriched 3aa (Fig. 2). It is worth mentioning that despite much exploration of the use of chiral NHCs, there has been less reports with nickel-catalyzed reactions ${ }^{31-40}$. $N$-(2-Biphenyl)- $\left(\mathbf{L}^{\star} \mathbf{1}\right)$ and $N$-(2-isopropylphenyl)- ( $\left.\mathbf{L}^{\star} \mathbf{2}\right)$ substituted $\mathrm{NHCs}^{41}$ were ineffective to give 3aa. In a similar manner, $N$-(2,7-diisopropylnaphthyl)- $\left(\mathbf{L}^{\star} \mathbf{4}\right)^{42}$ and $N$-(2,7-dicyclohexylnaphthyl) $\left(\mathbf{L}^{\star} 5\right)^{34}$-substituted NHCs also failed to yield any products. However, N-2,6-diethylphenyl-substituted NHC $\left(\mathbf{L}^{\star} \mathbf{3}\right)^{43}$ successfully gave hydronaphtho[1,8-bc]furan $3 \mathbf{a a}$ in $13 \%$ yield with high enantioselectivity $\left(94 \%\right.$ ee). NHCs $\mathbf{L}^{\star} \mathbf{6}^{35}$ and $\mathbf{L}^{\star} 7^{34}$ furnished 3aa in moderate chemical yields (36 and $48 \%$, respectively) with excellent enantioselectivities (98 and $89 \%$ ee, respectively). Given the excellent enantioselectivity with $\mathbf{L}^{\star} \mathbf{6}$, extensive effort was devoted to improving the yield. When the reaction was conducted at $60^{\circ} \mathrm{C}$ using a lower concentration $(0.02 \mathrm{M}$ of $\mathbf{2 a})$, 3aa was obtained in $74 \%$ yield with $98 \%$ enantioselectivity (see Supplementary Table 1 for detail).

Substrate scope. With the aforementioned optimal reaction conditions, we explored the scope of substrates (Fig. 3). A range of electron-rich and electron-deficient aryl-substituted enones 2 was examined with $\mathbf{1 a}$. The reaction proceeded smoothly with $\mathbf{2 b}$ and $\mathbf{2 c}$, giving $\mathbf{3 a b}$ and $\mathbf{3 a c}$ in 73 and $72 \%$ yields, respectively, with enantioselectivities of $98 \%$ each. Reaction with an enone containing 2 -furyl group (2d) was also examined with 1a to afford 3ad in $72 \%$ yield with $99 \%$ ee. In contrast, the reaction of 1a with 1-aryl-2-buten-1-ones (2e-2i) gave 3ae-3ai in slightly lower yields (62-70\%), albeit enantioselectivities remained excellent $(94-97 \%$ ee). In these cases, about $5 \%$ of fully- intermolecular $[2+2+2]$ cycloaddition products $\left(3^{\prime}\right)$ of an alkyne unit of dienone-yne (1a) with two enones $(\mathbf{2 e}-\mathbf{2} \mathbf{i})$ were observed (See Supplementary Fig. 10 for 3ae'). When ethyl group at $\mathrm{R}^{3}$ of an enone was introduced, a complex mixture was obtained. Next, alkynyl-cyclohexadienone substrates (1) were investigated by varying the substituents $\mathrm{R}^{1}$ and $\mathrm{R}^{2}$. Ethyl as well as 2-methoxyethyl-substituted alkynyl-cyclohexadienone (1 $\mathbf{b}$ and 1c) gave $3 \mathbf{b b}, 3 \mathbf{b j}$, $3 \mathbf{c a}$, and $3 \mathbf{c k}$ with $\mathbf{2 b}, \mathbf{2 j}$, $2 \mathbf{a}$, and $2 \mathbf{k}$, respectively in good yields $(60-73 \%)$ with excellent enantioselectivities (96-99\%). Aryl and alkyl groups on alkynes were also examined. Phenyl-acetylene-substituted dienone 1d gave 3de in good yield with excellent enantioselectivity (70\% yield and 99\% ee). Electron-rich anisyl-group substrate 1e gave 3 ec $(71 \%$ yield and $98 \%$ ee) and 3ek (68\% yield and 99\% ee) with $p$-halogenated ( $\mathrm{F}-$ and $\mathrm{Cl}-$ ) chalcones $\mathbf{2 c}$ and $\mathbf{2 k}$, respectively. No corresponding dehalogenated products were detected. An alkynyl-dienone if bearing an electron-deficient $p-\mathrm{CO}_{2} \mathrm{EtC}_{6} \mathrm{H}_{4}$ - group gave $3 \mathrm{fc}$ with $2 \mathrm{c}$ in $60 \%$ yield with $98 \%$ ee, whereas 3 ge was obtained in $71 \%$ yield with $96 \%$ ee from $p-\mathrm{CF}_{3}$-substituted $\mathbf{1 g}$. An ethyl-group and a triethylsilyloxy-methyl-substituted alkynyl-cyclohexadienone (1h and $\mathbf{1 i}$, respectively) gave corresponding tricyclic fused rings 3 he, 3ic, and $3 \mathbf{i k}$ with $2 \mathbf{e}, 2 \mathbf{c}$, and $2 \mathbf{k}$, respectively, in good yields and enantioselectivities (69-77\% yields, $95-98 \%$ ee). A $N$-tosyl analog of alkynyl-cyclohexadienone $\mathbf{1 j}$ failed to give the desired product $3 \mathbf{j c}$ with $\mathbf{2 c}$ under the present reaction conditions. This could have been due to coordination ability of tosyl group to nickel which inhibits the coordination of $2 \mathrm{c}^{44}, 45$. The absolute configurations of all five chiral centers in 3 were assigned according to an analogy with 3ik, which was unambiguously determined by X-ray crystallographic analysis (Fig. 3). It also supports all the stereo selective and regioselective outcomes in 3. 


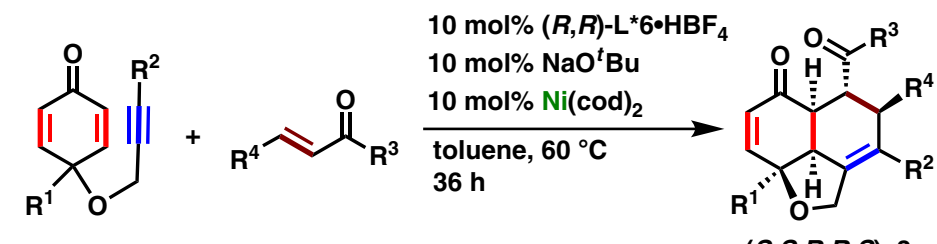

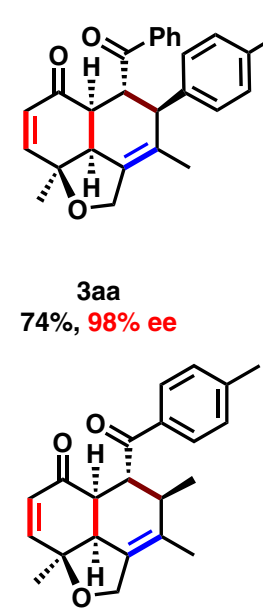

3ag
$67 \%, 95 \%$ ee
1<smiles>CC1=C2CO[C@]3(C)C=CC(=O)[C@@](C(=O)c4ccccc4)(C2)[C@H](c2ccc(F)cc2)C13</smiles><smiles>CC1=C2CO[C@@]3(C)C=CC(=O)[C@](C(=O)c4ccccc4)([C@@H](c4ccccc4)C2=O)[C@]13C</smiles>

$3 a b$ $73 \%, 98 \%$ ee 3ac
$72 \%, 98 \%$ ee

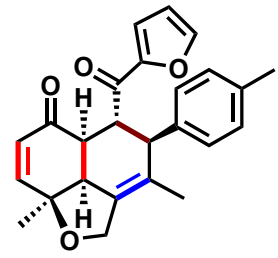

3ad $72 \%, 99 \%$ ee

$(S, S, R, R, S)-3$<smiles>CC1=C2CO[C@]3(C)C=CC(=O)[C@@](C(=O)c4ccc(F)cc4)(C23)[C@H]1C</smiles>

3ah $68 \%, 95 \%$ ee<smiles>CC1=C2CO[C@]3(C)C=CC(=O)[C@@]2(C3)[C@H](C(=O)c2cccc(F)c2)C1C</smiles>

3ai $70 \%, 97 \%$ ee

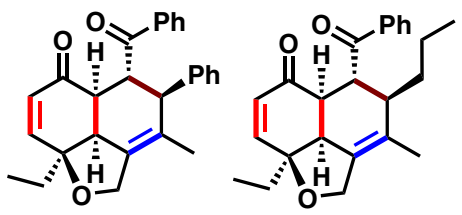

3bj $73 \%, 99 \%$ ee $\quad 65 \%, 96 \%$ ee<smiles>COc1ccc(C(=O)C2C(C)C(C)=C3CO[C@]4(C)C=CC(=O)[C@@H]2[C@@]34C)cc1</smiles>

3af $65 \%, 94 \%$ ee<smiles>COC[C@]12C=CC(=O)[C@]3(C(=O)c4ccc(Cl)cc4)C(c4ccc(Cl)cc4)C(C)=C(CO[C@@]13C)C2</smiles>

3ck $68 \% 98 \%$ ee

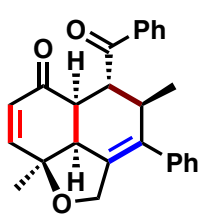

3de $70 \%, 99 \%$ ee<smiles></smiles>

3ec

$71 \%, 98 \%$ ee<smiles>COc1ccc(C2=C3CO[C@]4(C)C=CC(=O)[C@@]5(C3C(=O)c3ccccc3)[C@@H](c3ccc(Cl)cc3)C45C2)cc1</smiles><smiles>CCOC(=O)c1ccc(C2=C3CO[C@]4(C)C=CC(=O)[C@@](C)([C@H](C(=O)c5ccccc5)[C@H]4c4ccc(F)cc4)[C@@]32C)cc1</smiles>

3ek
$68 \%, 99 \%$ ee<smiles>C[C@@H]1C[C@]23C(=O)C=C[C@@](C)(OCC2=C1c1ccc(C(F)(F)F)cc1)[C@@H]3C(=O)c1ccccc1</smiles><smiles>CCC1=C2CO[C@]3(C)C=CC(=O)[C@@](C(=O)c4ccccc4)(C2C)[C@H]3[C@@H]1C</smiles>
$69 \%, 98 \%$ ee<smiles>COCC[C@]12OCC3=C(C)[C@@H](c4ccc(OC)cc4)[C@H](C(=O)P)[C@H](C(=O)C=CC1=O)[C@@]32C</smiles>

$3 \mathrm{ca}$

$60 \% 98 \%$ ee

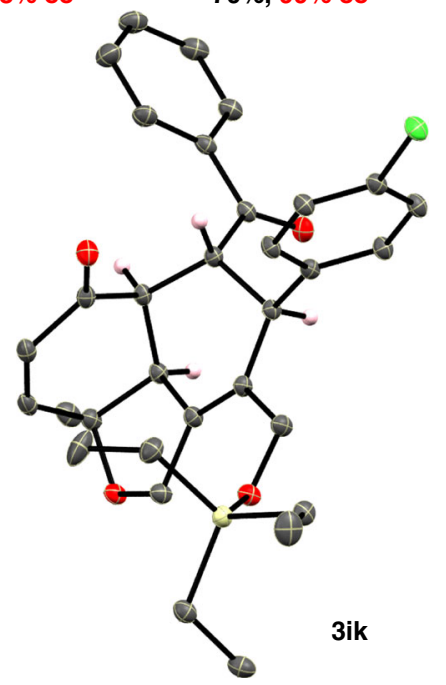

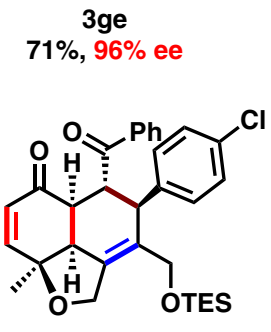

3ik

$77 \%$ 97\% ee

$3 f \mathrm{fc}$
$60 \%, 98 \%$ ee

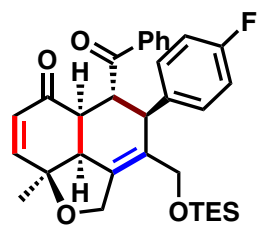

3ic

$74 \%$ 95\% ee

Fig. 3 Substrate Scope. Reaction was examined at 0.1-0.4 mmol scale. Isolated yields are given and enantioselectivity was determined by SFC equipped with a chiral stationary phase. ORTEP diagram of 3ik is shown with thermal ellipsoid at $30 \%$ probability level. Hydrogen atoms are omitted for clarity except those bound to the chiral carbon centers. Flack parameter for 3ik $=0.017(7)$

Gram scale synthesis and transformations of product $3 \mathrm{aa}$. To demonstrate applicability, a half-gram-scale reaction of $\mathbf{1 a}(0.53 \mathrm{~g}$, $3.0 \mathrm{mmol})$ was carried out with $2 \mathbf{a}(0.6 \mathrm{~g}, 2.5 \mathrm{mmol})$ to afford $3 \mathbf{a a}$ in $73 \%$ yield and $98 \%$ ee. These enantioenriched tricyclic products could be useful synthetic intermediates for further transformations (Fig. 4). The methylene group of a tetrahydrofuran ring was oxidized with $\mathrm{PCC}^{46}$ to yield a butyrolactone scaffold $(4,90 \%$ yield), that is present in natural products and also is a key synthetic intermediate in many sesquiterpenoids (Fig. 1a) ${ }^{6-9}$. Epoxidation and Michael addition of an enone gave the corresponding functionalized products $\mathbf{5}$ and $\mathbf{6}$ as single diastereomers in 75 and $85 \%$ yields, respectively, whereas hydrogenation of an 


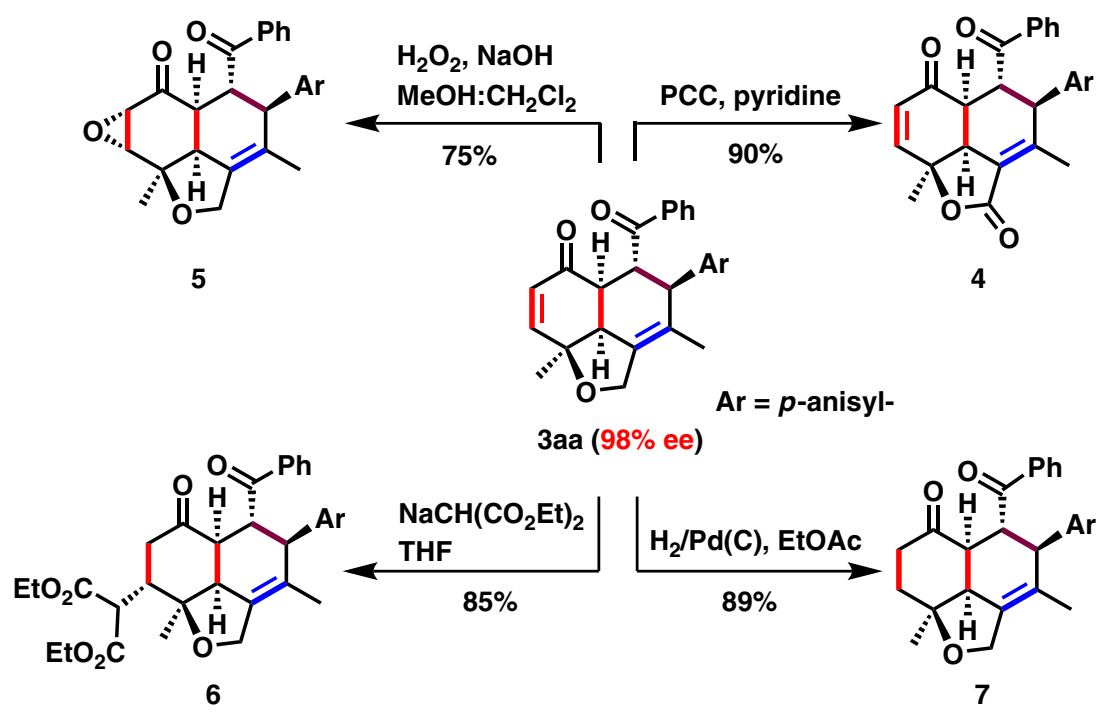

Fig. 4 Synthetic Transformation of 3aa. Isolated yields are given and enantioselectivity was determined by SFC equipped with a chiral stationary phase

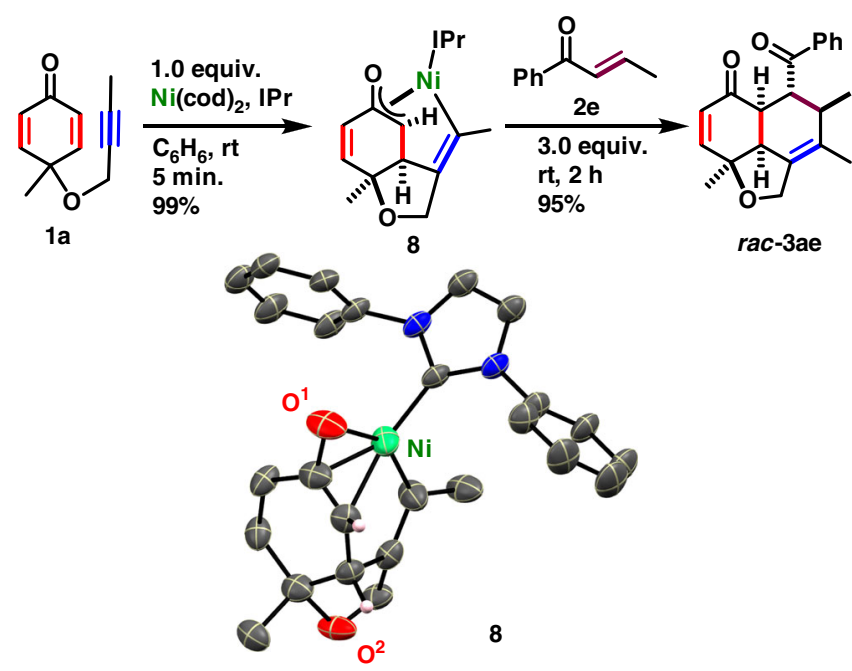

Fig. 5 Stoichiometric reaction with 1a. Isolated yield of $\mathbf{8}$ and rac-3ae are given. The molecular structure of $\eta^{3}$-cyclohexadienyl Ni-complex $\mathbf{8}$ is disordered and one of the disordered structure is depicted for simplicity with thermal ellipsoid at 30\% probability level. ${ }^{\text {P }}$ Pr groups and hydrogen atoms (except those bound to the chiral carbons) are omitted for clarity

enonic $\mathrm{C}=\mathrm{C}$ bond with $\mathrm{H}_{2} / \mathrm{Pd}(\mathrm{C})$ in ethyl acetate gave 7 in $89 \%$ yield. The enantioselectivity remained consistent in all these transformations.

Stoichiometric experiment. In order to gain deeper insight into a possible reaction mechanism, stoichiometric experiments were conducted in $\mathrm{C}_{6} \mathrm{D}_{6}$. An attempts to isolate a chiral nickelacycle corresponds to $\mathbf{1 g}$ using an optimal $\mathrm{NHC} \mathbf{L}^{\star} \mathbf{6}$ and $\mathrm{Ni}(\operatorname{cod})_{2}$ was unsuccessful. ${ }^{19} \mathrm{~F}$ NMR spectra showed seven peaks, revealed the existence of several intermediates, in which one of them might be much more reactive leads to desired product in the presence of an enone. However, an $\eta^{3}$-oxaallyl nickelacycle $(8)$ was isolated in $99 \%$ yield when a stoichiometric reaction of 1 a was conducted with $\mathrm{IPr}$ and $\mathrm{Ni}(\mathrm{cod})_{2}$. The molecular structure of $\mathbf{8}$ was confirmed by X-ray crystallography (Fig. 5). The ${ }^{1} \mathrm{H},{ }^{13} \mathrm{C}$, and $2 \mathrm{D}$ NMR analyses of $\mathbf{8}$ demonstrated that its structure in solution was consistent with that observed in crystal lattice. The reaction of $\mathbf{8}$ with 2 e gave rac-3ae in 95\% isolated yield, which supported that desymmetrization by oxidative cyclization would play a key role in the present transformation.

A plausible reaction mechanism is drawn on the basis of the results of the stoichiometric experiment and previous reports (Fig. 6) ${ }^{20-30,33-42}$. First, the intramolecular oxidative cyclization of 1 via the simultaneous coordination of an alkyne and an enantiotropic enone to the chiral $\mathrm{Ni}(0) / \mathbf{L}^{\star}$ species gives a desymmetrized nickelacycle $\mathbf{A}$, which would be in equilibrium with its $\eta^{3}$-oxaallylnickel structure $\mathbf{A}^{\prime}$. Coordination of $\mathbf{2}$ to nickel center of $\mathbf{A}$ giving $\mathbf{B}$, followed by insertion through a $\mathrm{Ni}-\mathrm{C}_{s p}{ }^{2}$ bond could form a thermodynamically favorable $\eta^{3}$-oxaallylnickel structure either $\mathbf{C}$ or $\mathbf{C}^{\prime}$. Then, a subsequent reductive elimination could afford a tricyclic fused structure $\mathbf{3}$ as a single diastereomer with the regeneration of nickel $(0)$ species.

In conclusion, a catalytic enantioselective method has been developed for the rapid construction of hydronaphtho[1,8-bc] furans with five contiguous chiral centers via desymmetrization of alkynyl-cyclohexadienone and formal $[4+2]$ cycloaddition reaction with nickel. The synthetic utility of tricyclic products was also demonstrated. Isolation of desymmetrized $\eta^{3}$-oxaallyl nickelacycle and subsequent reactions in the stoichiometric experiment revealed that desymmetrization by oxidative cyclization is the key in this transformation. Furthermore, unusual regioselectivity in the insertion step of enone revealed the diverse reactivity of an $\eta^{3}$-oxaallyl nickel-complex. The developed strategy involving two steps from the easily accessible phenols, demonstrates a practical and step economic protocol to access synthetically valuable fused tricyclic frameworks bearing five consecutive chiral carbon centers with excellent enantioselectivities.

\section{Methods}

General procedure for tricyclic product 3. To a screw cap vial in a glove box was added $\mathbf{L}^{*} \mathbf{6} \cdot \mathrm{HBF}_{4}(10 \mathrm{~mol} \%)$ and $\mathrm{NaO}^{t} \mathrm{Bu}(10 \mathrm{~mol} \%)$ and toluene $(5 \mathrm{ml})$. The suspension was allowed to stir at room temperature for $10 \mathrm{~min}$ and then $\mathrm{Ni}(\operatorname{cod})_{2}(10$ mol\%) was added. After further stirring for $10 \mathrm{~min}$ at room temperature was added a solution of alkynyl-cyclohexadienone $(1,0.24 \mathrm{mmol})$ and enone $(2,0.20 \mathrm{mmol})$ in toluene $(5 \mathrm{ml})$. The reaction mixture was taken out of glove box and heated at $60^{\circ}$ $\mathrm{C}$ for $36 \mathrm{~h}$ with stirring. After cooling to room temperature, the mixture was filtered through celite and washed with $\mathrm{Et}_{2} \mathrm{O}$. The filtrate was concentrated in vacuo and the residue was purified by silica gel flash chromatography (5-20\% ethyl acetate in hexane) to afford the desired product 3 . 


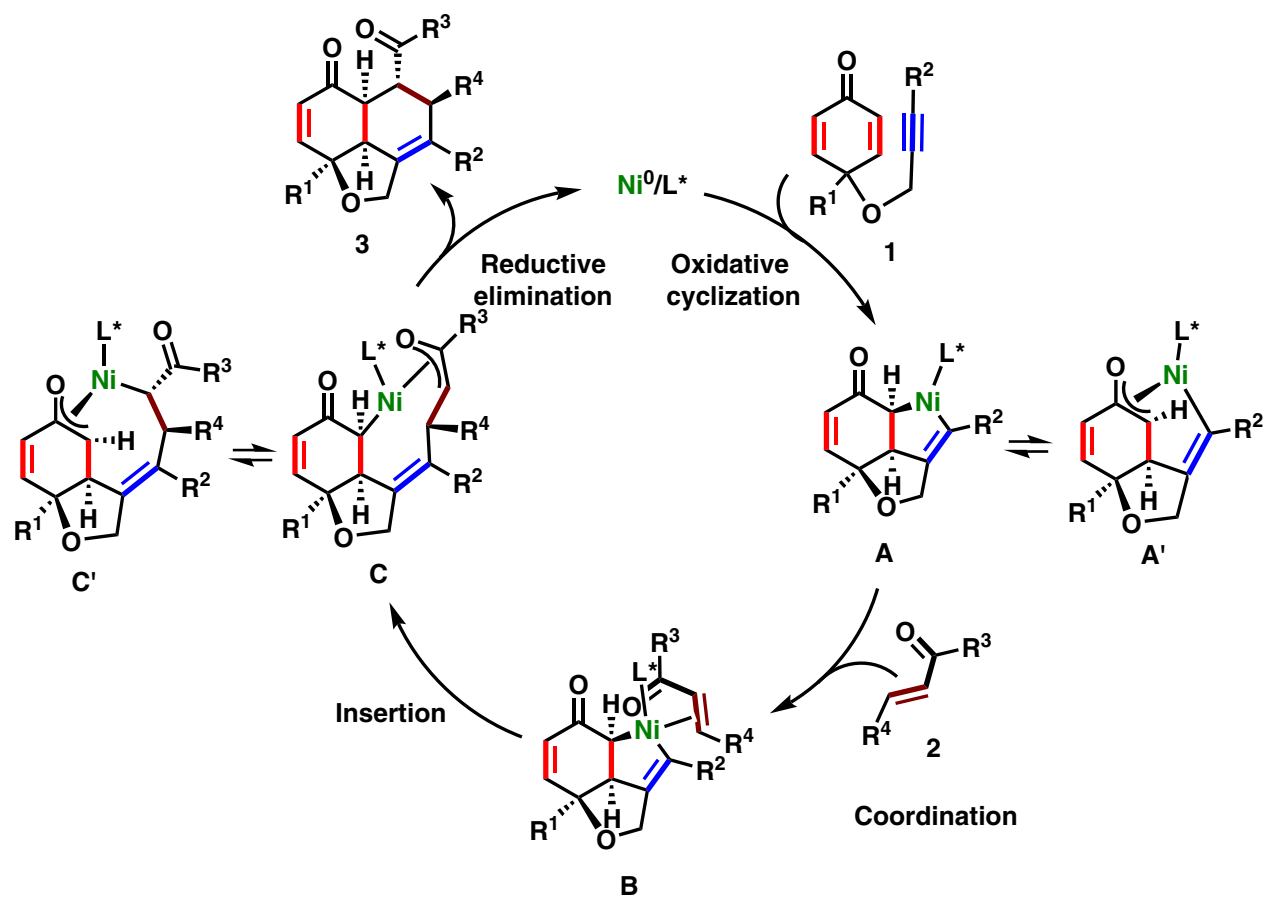

Fig. 6 A plausible reaction mechanism. Nickelacycle $\mathbf{A}$ is generated by enantioselective desymmetrization of $\mathbf{1}$ via oxidative cyclization. Subsequent insertion of an enone $\mathbf{2}$ to $\mathbf{A}$ gives a tricyclic product $\mathbf{3}$

Data availability. The X-ray crystallographic coordinates for structures reported in this study have been deposited at the Cambridge Crystallographic Data Centre (CCDC) under deposition numbers CCDC 1523827 (3ik) and 1523828 (8). These data can be obtained free of charge from The CCDC via www.ccdc.cam.ac.uk/ data_request/cif. All other data supporting the findings of this study are available within the article and its Supplementary Information file or from the authors upon reasonable request. For NMR spectra of the compounds in this article, see Supplementary Figs. 2-31.

Received: 11 February 2017 Accepted: 26 April 2017

Published online: 26 June 2017

\section{References}

1. Germain, J. \& Deslongchamps, P. Total synthesis of ( \pm -momilactone A. J. Org. Chem. 67, 5269-5278 (2002).

2. Liu, X. \& Lee, C.-S. Total synthesis of (-)-teucvidin. Org. Lett. 14, 2886-2889 (2012).

3. Jauch, J. et al. Total synthesis of azadirachtin-finally completed after 22 years. Angew. Chem. Int. Ed. 47, 34-37 (2008).

4. Nicolaou, K. C., Lim, Y. H. \& Becker, J. Total synthesis and absolute configuration of the bisanthraquinone antibiotic BE-43472B. Angew. Chem. Int. Ed. 48, 3444-3448 (2009).

5. Schütz, J. et al. Synthesis and biological evaluation of 14-alkoxymorphinans. 17. highly $\delta$ opioid receptor selective 14 -alkoxy-substituted indolo- and benzofuromorphinans. J. Med. Chem. 45, 5378-5383 (2002).

6. Ye, H., Deng, G., Liu, J. \& Qiu, F. G. Expedient construction of the Ziegler intermediate useful for the synthesis of forskolin via consecutive rearrangements. Org. Lett. 11, 5442-5444 (2009).

7. Leclaire, M., Levet, R., Péricaud, F., Ricard, L. \& Lallemand, J. Y. Studies on the preparation of the "Ziegler intermediate", a key intermediate in the total synthesis of forskolin. Tetrahedron 52, 7703-7718 (1996).

8. Miyaoka, H., Yamanishi, M., Kajiwara, Y. \& Yamada, Y. Total synthesis of cladocorans A and B: a structural revision. J. Org. Chem. 68, 3476-3479 (2003).

9. Hanessian, S., Boyer, N., Reddy, G. J. \& Deschênes-Simard, B. Total synthesis of oidiodendrolides and related norditerpene dilactones from a common precursor: metabolites CJ-14,445, LL-Z1271 $\gamma$, oidiolactones A, B, C, and D, and nagilactone F. Org. Lett. 11, 4640-4643 (2009).

10. Trost, B. M. et al. The atom economy-a search for synthetic efficiency. Science 254, 1471-1477 (1991).

11. Lautens, M., Klute, W. \& Tam, W. Transition metal-mediated cycloaddition reactions. Chem. Rev. 96, 49-92 (1996).
12. Malacria, M. et al. Selective preparation of complex polycyclic molecules from acyclic precursors via radical mediated- or transition metal-catalyzed cascade reactions. Chem. Rev. 96, 289-306 (1996).

13. Trost, B. M. \& Krische, M. J. Transition metal catalyzed cycloisomerizations. Synlett. 1998, 1-16 (1998).

14. Martín-Santos, C. et al. Highly enantioselective construction of tricyclic derivatives by the desymmetrization of cyclohexadienones. Angew. Chem. Int. Ed. 53, 8184-8189 (2014).

15. Kalstabakken, K. A. \& Harned, A. M. Asymmetric transformations of achiral 2,5-cyclohexadienones. Tetrahedron 70, 9571-9585 (2014).

16. Zeng, X.-P., Cao, Z.-Y., Wang, Y.-H., Zhou, F. \& Zhou, J. Catalytic enantioselective desymmetrization reactions to all-carbon quaternary stereocenters. Chem. Rev. 116, 7330-7396 (2016).

17. He, Z.-T. et al. Efficient access to bicyclo[4.3.0]nonanes: copper-catalyzed asymmetric silylative cyclization of cyclohexadienone-tethered allenes. Angew. Chem. Int. Ed. 54, 14815-14818 (2015).

18. Fukui, Y. et al. Tunable arylative cyclization of 1,6-enynes triggered by rhodium (III)-catalyzed C-H activation. J. Am. Chem. Soc. 136, 15607-15614 (2014).

19. Clarke, C., Incerti-Pradillos, C. A. \& Lam, H. W. Enantioselective nickelcatalyzed anti-carbometallative cyclizations of alkynyl electrophiles enabled by reversible alkenylnickel $E / Z$ isomerization. J. Am. Chem. Soc. 138, 8068-8071 (2016).

20. Tasker, S. Z., Standley, E. A. \& Jamison, T. F. Recent advances in homogeneous nickel catalysis. Nature 509, 299-309 (2014).

21. Ohashi, M., Hoshimoto, Y. \& Ogoshi, S. Aza-nickelacycle key intermediate in nickel(0)-catalyzed transformation reactions. Dalton Trans. 44, 12060-12073 (2015).

22. Hoshimoto, Y., Ohashi, M. \& Ogoshi, S. Catalytic transformation of aldehydes with nickel complexes through $\eta^{2}$ coordination and oxidative cyclization. Acc. Chem. Res. 48, 1746-1755 (2015).

23. Standley, E. A., Tasker, S. Z., Jensen, K. L. \& Jamison, T. F. Nickel catalysis: synergy between method development and total synthesis. Acc. Chem. Res. 48, 1503-1514 (2015).

24. Jackson, E. P. et al. Mechanistic basis for regioselection and regiodivergence in nickel-catalyzed reductive couplings. Acc. Chem. Res. 48, 1736-1745 (2015).

25. Seo, J., Chui, H. M. P., Heeg, M. J. \& Montgomery, J. Novel chemoselectivity and stereochemical aspects of nickel-catalyzed $[2+2+2]$ cycloadditions. J. Am. Chem. Soc. 121, 476-477 (1999).

26. Montgomery, J. \& Savchenko, A. V. Nickel-catalyzed cyclizations of alkynyl enones with concomitant stereoselective tri- or tetrasubstituted alkene introduction. J. Am. Chem. Soc. 118, 2099-2100 (1996).

27. Montgomery, J., Oblinger, E. \& Savchenko, A. V. Nickel-catalyzed organozincpromoted carbocyclizations of electron-deficient alkenes with tethered unsaturation. J. Am. Chem. Soc. 119, 4911-4920 (1997). 
28. Chevliakov, M. V. \& Montgomery, J. A stereodivergent approach to (-)- $\alpha-$ kainic acid and (+)- $\alpha$-allokainic acid utilizing the complementarity of alkyne and allene cyclizations. J. Am. Chem. Soc. 121, 11139-11143 (1999).

29. Ogoshi, S., Nishimura, A. \& Ohashi, M. Nickel-catalyzed [2+2+2] cycloaddition of two enones and an alkyne. Org. Lett. 12, 3450-3452 (2010).

30. Nishimura, A., Ohashi, M. \& Ogoshi, S. Nickel-catalyzed intermolecular $[2+2]$ cycloaddition of conjugated enynes with alkenes. J. Am. Chem. Soc. 134 15692-15695 (2012).

31. Kumar, R., Hoshimoto, Y., Yabuki, H., Ohashi, M. \& Ogoshi, S. Nickel(0)catalyzed enantio- and diastereoselective synthesis of benzoxasiloles: ligandcontrolled switching from inter- to intramolecular aryl-transfer process. J. Am. Chem. Soc. 137, 11838-11845 (2015).

32. Kumar, R. et al. Nickel $(0) / N$-heterocyclic carbene-catalyzed asymmetric $[2+2+2]$ cycloaddition of two enones and an alkyne: Access to cyclohexenes with four contiguous stereogenic centers. Org. Lett. 17, 6018-6021 (2015).

33. Kumar, R. et al. Nickel-catalyzed enantioselective synthesis of cyclobutenes via $[2+2]$ cycloaddition of $\alpha, \beta$-unsaturated carbonyls with 1,3-enynes. Synthesis $\mathbf{4 8}$, 2789-2794 (2016).

34. Hayashi, Y., Hoshimoto, Y., Kumar, R., Ohashi, M. \& Ogoshi, S. Nickel(0)catalyzed intramolecular reductive coupling of alkenes and aldehydes or ketones with hydrosilanes. Chem. Commun. 52, 6237-6240 (2016).

35. Chaulagain, M. R., Sormunen, G. J. \& Montgomery, J. New N-heterocyclic carbene ligand and its application in asymmetric nickel-catalyzed aldehyde/ alkyne reductive couplings. J. Am. Chem. Soc. 129, 9568-9569 (2007).

36. Sato, Y., Hinata, Y., Seki, R., Oonishi, Y. \& Saito, N. Nickel-catalyzed enantioand diastereoselective three-component coupling of 1,3-dienes, aldehydes, and silanes using chiral $N$-heterocyclic carbenes as ligands. Org. Lett. 9, 5597-5599 (2007).

37. Ahlin, J. S. E., Donets, P. A. \& Cramer, N. Nickel(0)-catalyzed enantioselective annulations of alkynes and arylenoates enabled by a chiral NHC ligand: efficient access to cyclopentenones. Angew. Chem. Int. Ed. 53, 13229-13233 (2014).

38. Donets, P. A. \& Cramer, N. Ligand-controlled regiodivergent nickel-catalyzed annulation of pyridones. Angew. Chem. Int. Ed. 54, 633-637 (2015).

39. Ahlin, J. S. E. \& Cramer, N. Chiral $N$-heterocyclic carbene ligand enabled nickel (0)-catalyzed enantioselective three-component couplings as direct access to silylated indanols. Org. Lett. 18, 3242-3245 (2016).

40. Ho, C.-Y., Chan, C.-W. \& He, L. Catalytic asymmetric hydroalkenylation of vinylarenes: electronic effects of substrates and chiral $N$-heterocyclic carbene ligands. Angew. Chem. Int. Ed. 54, 4512-4516 (2015).

41. Seiders, T. J., Ward, D. W. \& Grubbs, R. H. Enantioselective rutheniumcatalyzed ring-closing metathesis. Org. Lett. 3, 3225-3228 (2001).

42. Luan, X. et al. Matching the chirality of monodentate $N$-heterocyclic carbene ligands: a case study on well-defined palladium complexes for the asymmetric $\alpha$-arylation of amides. Org. Lett. 10, 5569-5572 (2008).

43. Matsumoto, Y., Yamada, K. \& Tomioka, K. $C_{2}$ symmetric chiral NHC ligand for asymmetric quaternary carbon constructing copper-catalyzed conjugate addition of Grignard reagents to 3-substituted cyclohexenones. J. Org. Chem. 73, 4578-4581 (2008).

44. Ogoshi, S., Ikeda, H. \& Kurosawa, H. Formation of an aza-nickelacycle by reaction of an imine and an alkyne with nickel(0): oxidative cyclization, insertion, and reductive elimination. Angew. Chem. Int. Ed. 46, 4930-4932 (2007).

45. Hoshimoto, Y., Ohata, T., Sasaoka, Y., Ohashi, M. \& Ogoshi, S. Nickel(0)catalyzed $[2+2+1]$ carbonylative cycloaddition of imines and alkynes or norbornene leading to $\gamma$-lactams. J. Am. Chem. Soc. 136, 15877-15880 (2014). 46. Bonadies, F. \& Bonini, C. Oxidation of active methylene compounds by pyridinium chlorochromate. Synth. Commun. 18, 1573-1580 (1988).

\section{Acknowledgements}

This work was supported by a Grant-in-Aid for Scientific Research (A) (No. JP25708018) and (B) (No. JP15K17824), Scientific Research on Innovative Areas (Nos. JP15H00943 and JP15H05803) from MEXT and by JST, Advanced Catalytic Transformation Program for Carbon Utilization (ACT-C, Grant Number JPMJCR12Y6), Japan. Y.H. acknowledges support from the Frontier Research Base for Global Young Researchers, Osaka University, on the program of MEXT. We sincerely thank Prof. Dr. Norimitsu Tohnai, Graduate School of Engineering, Osaka University, Japan for collecting X-ray data of 3ik. We also thank Prof. Dr. Mary Grellier, Laboratoire de Chimie de Coordination, CNRS, Toulouse, France for discussion on mechanistic part.

\section{Author contributions}

S.O. and R.K. conceived and designed the synthetic routes. R.K. and Y.H. prepared the manuscript, and edited by all other authors. R.K. and E.T. carried out the experiments. M.O. analyzed the X-ray data.

\section{Additional information}

Supplementary Information accompanies this paper at doi:10.1038/s41467-017-00068-8

Competing interests: The authors declare no competing financial interests.

Reprints and permission information is available online at http://npg.nature.com/ reprintsandpermissions/

Publisher's note: Springer Nature remains neutral with regard to jurisdictional claims in published maps and institutional affiliations.

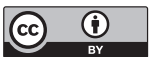

Open Access This article is licensed under a Creative Commons Attribution 4.0 International License, which permits use, sharing, adaptation, distribution and reproduction in any medium or format, as long as you give appropriate credit to the original author(s) and the source, provide a link to the Creative Commons license, and indicate if changes were made. The images or other third party material in this article are included in the article's Creative Commons license, unless indicated otherwise in a credit line to the material. If material is not included in the article's Creative Commons license and your intended use is not permitted by statutory regulation or exceeds the permitted use, you will need to obtain permission directly from the copyright holder. To view a copy of this license, visit http://creativecommons.org/ licenses/by/4.0/

(c) The Author(s) 2017 